\title{
Treatment of Refractory Mastocytic Enterocolitis with Budesonide
}

\author{
Merin Elizabeth Kuruvilla ${ }^{1}$, Sonia Mathew ${ }^{2}$, Vaidehi Avadhani ${ }^{3}$
}

1) Department of Allergy/

Immunology, Emory

University, Atlanta, Georgia;

2) Mercer University School of

Medicine, Macon, Georgia;

3) Department of Pathology,

Emory University, Atlanta,

Georgia, USA

\author{
Address for correspondence: \\ Merin Elizabeth Kuruvilla \\ Department of Allergy/ \\ Immunology \\ Emory University \\ 1365 Clifton Rd NE, Atlanta, \\ GA-30322, USA \\ merin.ek1@gmail.com
}

\begin{abstract}
Mast cells (MCs) are being increasingly implicated as a possible contributor to symptoms in diarrhea predominant irritable bowel syndrome (IBS). The term "mastocytic enterocolitis" was proposed to describe an increase in mucosal MCs in patients with chronic diarrhea due to functional gastrointestinal disease (FGID). The efficacy of anti-MC mediator therapy (antihistamines and MC stabilizers) has been well documented in this setting. Here we describe the treatment with oral budesonide of mastocytic enterocolitis refractory to standard anti-MC therapy.
\end{abstract}

Key words: mast cells - irritable bowel syndrome - mastocytic enterocolitis - antihistamines - mast cell stabilizer - budesonide.

Abbreviations: FGID: functional gastrointestinal disease; hpf: high power field; IBS: irritable bowel syndrome; MCs: mast cells; SM: systemic mastocytosis.

\section{INTRODUCTION}

Mast cells (MCs) are being increasingly implicated as a possible contributor to symptoms in diarrhea predominant irritable bowel syndrome (IBS). The term "mastocytic enterocolitis" was proposed by Jakate et al. [1] to describe an increase in mucosal MCs in patients with chronic diarrhea due to functional gastrointestinal disease (FGID). The efficacy of anti-MC mediator therapy (antihistamines and MC stabilizers) has been well documented in this setting [1-4]. Here we describe the treatment with oral budesonide of mastocytic enterocolitis refractory to standard anti-MC therapy.

\section{CASE REPORT}

Our patient is a 39 -yearold man who suffered from chronic constipation alternating with diarrhea for several years, suggestive of IBS. He had no other cutaneous signs or systemic symptoms. Five months prior to presentation, symptoms acutely worsened, with intermittent sharp abdominal pain, bloating, and diarrhea occurring up to eight times per day. There were no identifiable food triggers, or evidence of infectious gastroenteritis. Empirical dietary eliminations had been unsuccessful at ameliorating symptoms. In addition, he had a history of allergic rhinitis, Asperger syndrome and epilepsy.

Computerized tomography with contrast showed a fatty pancreas and mildly dilated small bowel concerning for enteritis. Colonoscopy did not detect any lesions. Histology examination depicted significant associated tissue eosinophilia (Fig. 1). Mast cells tryptase immunostaining found increased MC infiltration (up to 25 per high power field, hpf) within the lamina propria (Fig. 2). Mast cells aggregates and atypical morphology such as spindle-shaped cells were not observed. CD25 co-expression was not evaluated.

Baseline tryptase and serum IgE levels were normal at 3.3 $\mathrm{ng} / \mathrm{ml}$ and $66 \mathrm{IU} / \mathrm{ml}$, respectively. Specific IgE to aeroallergens were tested due to rhinitis symptoms, and were elevated only to grass pollen. Evaluation for the c-KIT mutation was not pursued since the patient failed to meet major or other minor criteria for systemic mastocytosis and had a REMA score of 1 indicating low risk for MC clonality. The REMA score is a validated score used to predict MC clonality with a minimum threshold score of 2 [5].

The patient was diagnosed with mastocytic enterocolitis, and we initiated a regimen of cetirizine $10 \mathrm{mg}$ twice daily, 


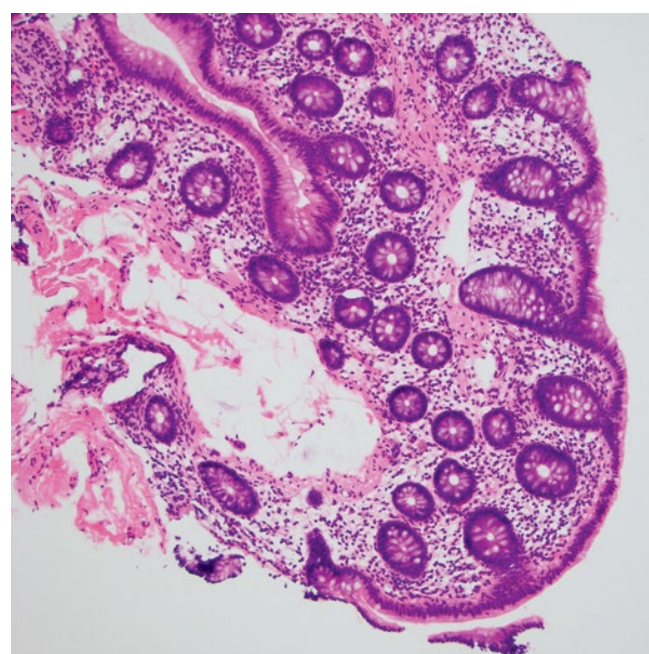

Fig. 1. Colonic mucosa with mild increased eosinophils and scattered mast cells. H\&E stain, 200x.

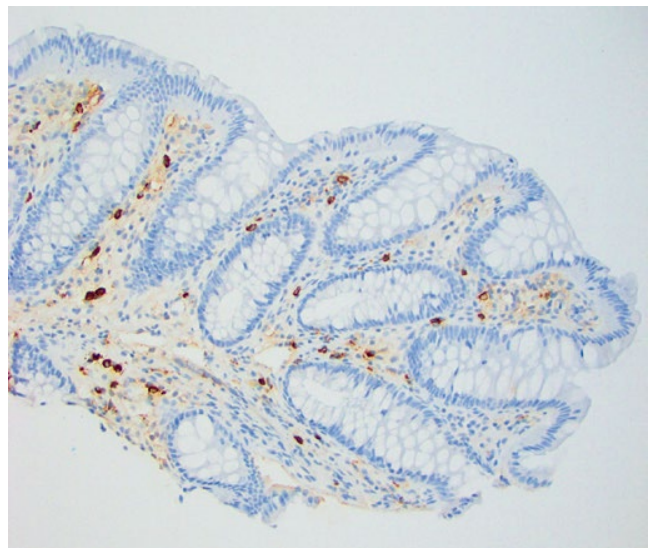

Fig. 2. Colonic mucosa with tryptase immunostain highlighting the increased mast cells. Tryptase IHC 200x.

and famotidine $20 \mathrm{mg}$ twice daily. He reported some but not substantial improvement. The addition of oral cromolyn (200 mg prior to meals and at bedtime, up to four times daily) afforded further but incomplete relief. He continued to have episodes of abdominal cramps and diarrhea a couple of times daily. A trial of oral budesonide $3 \mathrm{mg}$ three times daily was subsequently instituted, resulting in rapid and near complete resolution of abdominal pain and diarrhea output. Attempts to wean oral antihistamines were associated with mild symptom recurrence. Therefore, the patient is currently maintained on oral budesonide in conjunction with antihistamines and cromolyn sodium, and has no active symptoms.

\section{DISCUSSION}

Gastrointestinal mucosal MCs are key regulators of intestinal sensory and motor function. They have long been implicated in the pathogenesis of FGIDs, particularly in diarrhea predominant IBS [6]. Their activation and released mediators appear to contribute to the development of abdominal pain and diarrhea. A substantial increase in activated MCs releasing histamine has been observed in proximity to nerves that are correlated with abdominal pain in IBS [7] Histamine has been shown to sensitize enteric afferents, causing visceral hypersensitivity. This has supported the role of therapeutic MC blockade with $\mathrm{H} 1$ and $\mathrm{H} 2$ blockers in FGIDs [1-4]. The MC stabilizer ketotifen was also found to significantly decrease abdominal pain and other symptoms in a prospective randomized study of IBS patients [8]. Therefore, mastocytic enterocolitis is not intended to specify a distinct diagnosis, but rather denotes potential benefit from anti-MC therapies.

Jakate et al. [1] first coined the term "mastocytic enterocolitis" to describe this excess of gastrointestinal MCs in FGIDs. It was defined as the presence of greater than $20 \mathrm{MCs} /$ hpf in the gastrointestinal tract mucosa [1]. This cutoff value represented 2 standard deviations (SD) beyond the normal mean values for the colon and duodenum (13.6 and 13.2 MCs/ hpf, respectively). This recommendation was used, but it is based on relatively weak evidence, and a universally accepted cutoff for significant MC counts remains unknown.

In fact, other studies have found mild or no differences in the degree of MC infiltration in IBS [9]. Based on this variability, investigators have proposed that individual MC counts in patients with diarrhea predominant FGIDs are uninterpretable. They advocate empiric MC targeted therapy in patients with unremarkable routine histology [10].

Several reports document the efficacy of $\mathrm{H} 1$ and $\mathrm{H} 2$ blockers in treatment of FGIDs with excess gastrointestinal mucosal MCs. Mast cell stabilizers also seem to have some effect in such diseases; however, the effects are rather weak and inconsistent [8]. Add-on therapy with oral budesonide was chosen since it may conceivably address mucosal inflammation associated with MC activation that leads to gastrointestinal dysfunction. The drug was well tolerated and led to almost complete symptom resolution.

Our patient did not have evidence of systemic mastocytosis (SM), which presents with gastrointestinal symptoms in 60$80 \%$ of cases, most frequently abdominal pain and diarrhea. While the major criterion for diagnosis is the presence of more than $15 \mathrm{MCs} / \mathrm{hpf}$ in an extracutaneous organ, he did not have a clinical history suggestive of SM or fulfill any of the minor criteria tested (atypical MCs and elevated serum tryptase).

\section{CONCLUSION}

This case demonstrates the potential role of budesonide, a corticosteroid with a high mucosal activity and a low bioavailability, in treating FGIDs with a possible MC component. This therapy is worth further investigation for its utility in diarrhea-predominant IBS with or without counting gastrointestinal mucosal MCs.

Conflicts of interest: None to declare.

Authors' contributions: M.K. drafted the manuscript; S.M. reviewed the literature; V.A. pathology images and description.

\section{REFERENCES}

1. Jakate S, Demeo M, John R, Tobin M, Keshavarzian A. Mastocytic enterocolitis: increased mucosal mast cells in chronic intractable diarrhea. Arch Pathol Lab Med 2006;130:362-367. 
2. Seo H, Park SH, Byeon JS, et al. Chronic intractable diarrhea caused by gastrointestinal mastocytosis. Intest Res 2016;14:280-284. doi:10.5217/ ir.2016.14.3.280

3. Akhavein M A, Patel NR, Muniyappa PK, Glover SC. Allergic mastocytic gastroenteritis and colitis: an unexplained etiology in chronic abdominal pain and gastrointestinal dysmotility. Gastroenterol Res Pract 2012;2012:950582.doi:10.1155/2012/950582

4. Ogilvie-McDaniel C, Blaiss M, Osborn FD, Carpenter J. Mastocytic enterocolitis: a newly described mast cell entity. Ann Allergy Asthma Immunol 2008;101:645-646. doi:10.1016/S1081-1206(10)60231-1

5. Alvarez-Twose I, González-de-Olano D, Sánchez-Muñoz L, et al. Validation of the REMA score for predicting mast cell clonality and systemic mastocytosis in patients with systemic mast cell activation symptoms. Int Arch Allergy Immunol 2012;157:275-280. doi:10.1159/000329856

6. Wouters MM, Vicario M, Santos J. The role of mast cells in functional GI disorders. Gut 2016;65:155-168. doi:10.1136/gutjnl-2015-30915
7. Barbara G, Wang B, Stanghellini V, et al. Mast cell-dependent excitation of visceral-nociceptive sensory neurons in irritable bowel syndrome. Gastroenterology 2007;132:26-37. doi:10.1053/j. gastro.2006.11.039

8. Klooker TK, Braak B, Koopman KE, et al. The mast cell stabilizer ketotifen decreases visceral hypersensitivity and improves intestinal symptoms in patients with irritable bowel syndrome. Gut 2010;59:1213 1221. doi:10.1136/gut.2010.213108

9. Doyle LA, Sepehr GJ, Hamilton MJ, Akin C, Castells MC, Hornick JL. A Clinicopathologic study of 24 cases of systemic mastocytosis involving the gastrointestinal tract and assessment of mucosal mast cell density in irritable bowel syndrome and asymptomatic patients. Am J Surg Pathol 2014;38:832-843. doi:10.1097/PAS.0000000000000190

10. Sethi A, Jain D, Roland BC, et al. Performing colonic mast cell counts in patients with chronic diarrhea of unknown etiology has limited diagnostic use. Arch Pathol Lab Med 2015;139:225-232. doi:10.5858/ arpa.2013-0594-OA 